\title{
INDEX TO VOLUME 54
}

Author

ANdré, Debra A., Mullen,

GARY L. \& NiederReITER,

HARALD

Baur, Gerhard \&

SHAWYER, BRUCE

Bergé, A.-M., Martinet, J. \& Olivier, M.

Berger, Alan E.

Bernardi, Christine,

Debit, NAÏMA \&

MADAY, YvoN

BLECKSMITH, RICHARD,

BRILLHART, JOHN \&

GERST, IRVING

BOJANOV, BORISLAV \&

Nikolov, Geno

BorweIN, P. B. \&

LORING, T. A.

Bosma, WIEB

BRILlHART, JOHN

Bruaset, A. M., Tveito, A. \& WinTHER, R.

Buchmann, J., Sands, J. W. \& Williams, H. C.

BUfFONI, GIUSEPPE

Carnicer, J. \& Gasca, M.

Chou, S. H. \& Li, Q.

Chui, Charles K. \&

He, Tian-Xiao

Cockburn, Bernardo,

Hou, SuCHung \&

Shu, ChI-Wang

Cohen, Graeme L.

Coppersmith, Don

Debit, NaÏma

Delmer, Francine \&

Deshouillers, Jean-Marc

DenerT, M.

Deshouillers, Jean-Marc
Title

Page

Figures of merit for digital multistep pseudorandom num-

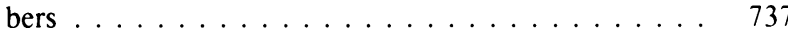

Best $L^{2}$-approximation of convergent moment series . .

The computation of sextic fields with a quadratic subfield

869

Accurate calculation of functions used in a model of the nematic behavior of self-assembling systems . . . . . .

Coupling finite element and spectral methods: First results . . . . . . . . . . . . . . . . .

On the mod 2 reciprocation of infinite modular-part products and the parity of certain partition functions . . . . . . . . . . . . . . . . . .

Comparison of Birkhoff type quadrature formulae . . . . .

Some questions of Erdös and Graham on numbers of the form $\sum g_{n} / 2^{g_{n}} . \ldots \ldots \ldots \ldots \ldots \ldots \ldots \ldots$

Approximation by mediants . . . . . . . . . . . 421

See: Blecksmith, Richard, Brillhart, John \& Gerst,

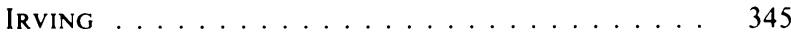

On the stability of relaxed incomplete LU factorizations . 701

$p$-adic computation of real quadratic class numbers $\ldots$.

855

Nonnegative and skew-symmetric perturbations of a matrix with positive inverse . . . . . . . . . . . .

Evaluation of multivariate polynomials and their deriva-

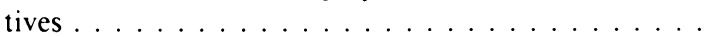

Convergence of the nonconforming Wilson element for a class of nonlinear parabolic problems . . . . . . . . . 509

Bivariate $C^{\prime}$ quadratic finite elements and vertex splines .

The Runge-Kutta local projection discontinuous Galerkin finite element method for conservation laws IV: The multidimensional case . . . . . . . . . . . . 545

On an integer's infinitary divisors . . . . . . . . . 395

Fermat's Last Theorem (case 1) and the Wieferich criterion 895

See: Bernardi, Christine, Debit, Naïma \& Maday, Yvon . . . . . . . . . . . . . . . . 21

On the computation of $g(k)$ in Waring's problem . . . 885

The genus zeta function of hereditary orders in central simple algebras over global fields . . . . . . . . . . . . . 449

Sec: Delmer, Francine \& Deshouillers, Jean-Marc . . 885 
Author

Duijvestijn, A. J. W. \& Helthuis, B.

Durán, Ricardo G. \& Nochetto, Ricardo $\mathrm{H}$.

EDIXHOVEN, BAS, DE Groot, ARNold \& TOP, JAAP

Elman, Howard C. \& Golub, Gene H.

Fishman, George S.

Gasca, M.

GERST, IRVING

Golub, Gene H.

DE GROOT, ARNOLD

Hanlon, Phil \&

WALES, DAVID

Hansbo, Peter

Hasegawa, Takemitsu,

TORII, TATSUO \&

Sugiura, Hiroshi

He, Tian-Xiao

Helthuis, B.

Hou, Suchung

Johnson, Claes,

SZEPESSY, ANDERS \&

Hansbo, Peter

JUST, BETTINA

Kvaernø, Anne

LI, Q.

LI, ZI-CAI \& Mathon, Rudolf

LORING, T. A.

LYNESS, J. N.

MADAY, YvoN

MADYCH, W. R. \&

Nelson, S. A.

MARTINET, J.

MATHON, RUdOLF

Montgomery, Peter L. \& Silverman, Robert D.

Mullen, Gary L.

NELSON, S. A.

NiederReiter, Harald \& SLOAN, IAN H.
Title

Page

Some new polyhedra with vertex degree 4 and/or 5 only

749

Weighted inf-sup condition and pointwise error estimates for the Stokes problem . . . . . . . . . .

Elliptic curves over the rationals with bad reduction at only one prime ..............

Iterative methods for cyclically reduced non-self-adjoint linear systems . . . . . . . . . . . . .

Multiplicative congruential random number generators with modulus $2^{\beta}$ : An exhaustive analysis for $\beta=32$ and a partial analysis for $\beta=48 \ldots \ldots \ldots$

See: Carnicer, J. \& Gasca, M. . . . . . . . . . . . .

See: Blecksmith, Richard, Brillhart, John \& Gerst,

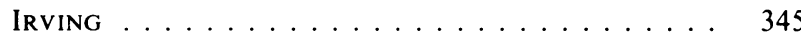

See: Elman, Howard C. \& Golub, Gene H. . . . . . . . 671

See: Edixhoven, BAS, DE Groot, ARnold \& Top, JAAP 413

Computing the discriminants of Brauer's centralizer algebras ..................... 771

See: Johnson, Claes, Szepessy, Anders \& Hansbo, Peter 107

An algorithm based on the FFT for a generalized Chebyshev interpolation ............... 195

See: Chui, Charles K. \& He, Tian-Xiao . . . . . . . . 169

See: Duijvestijn, A. J. W. \& Helthuis, B. . . . . . . . 749

See: Cockburn, Bernardo, Hou, Suchung \& Shu, ChiWANG .

On the convergence of shock-capturing streamline diffusion finite element methods for hyperbolic conservation laws . . . . . . . . . . . . . . 107

Integer relations among algebraic numbers . . . . . . . 467

Runge-Kutta methods applied to fully implicit differentialalgebraic equations of index $1 \ldots \ldots \ldots 58$

See: Chou, S. H. \& Li, Q. . . . . . . . . . . . . 509

Error and stability analysis of boundary methods for elliptic problems with interfaces............ 41

See: Borwein, P. B. \& Loring, T. A. . . . . . . . . . 377

See: Sloan, I. H. \& Lyness, J. N. . . . . . . . . . . . . . 649

See: Bernardi, Christine, Debit, Naïma \& Maday,

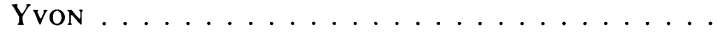

Multivariate interpolation and conditionally positive definite functions. II . . . . . . . . . . 211

See: Bergé, A.-M., Martinet, J. \& Olivier, M. . . . . 869

See: Li, Zi-Cai \& Mathon, Rudolf . . . . . . . . . 41

An FFT extension to the $P-1$ factoring algorithm . . . 839

See: André, Debra A., Mullen, Gary L. \& Nieder-

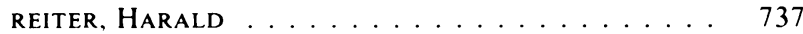

See: Madych, W. R. \& Nelson, S. A. . . . . . . . . 211

Lattice rules for multiple integration and discrepancy . . . 303 
Author

NiederReIter, HARALD

Nikolov, Geno

Nochetto, Ricardo $\mathrm{H}$.

Olivier, M.

Rabinowitz, Philip

RAHMAN, QAZI I. \& SCHMEISSER, GERHARD

Richter, GERARD R.

Rump, Siegfried M.

SANDS, J. W.

SCHMEISSER, Gerhard

SCOtT, L. RIDGWAY \& Zhang, Shangyou

Shawyer, Bruce

SHOUP, Victor

Shu, ChI-WaNG

Silverman, Robert D.

Sloan, I. H. \&

LYNESS, J. N.

SLOAN, IAN H. \&

WALSH, LiNDA

Sloan, IAN $\mathrm{H}$.

Stenberg, Rolf

Sugiura, Hiroshi

Suri, Manil

SZEPESSY, ANDERS

TaO, Tang

Teitelbaum, Jeremy

TOP, JAAP

TORII, TATSUO

Tsuchiya, TAKuYa

Tveito, A.

Wade, Bruce A.

Wales, David

WALSH, LINDA

WAN, DAQING
Title

Page

See: André, Debra A., Mullen, Gary L. \& Niederreiter, HARALD ................ 737

See: Bojanov, Borislav \& Nikolov, Geno . . . . . . 627

See: Durán, Ricardo G. \& Nochetto, Ricardo H. . . . 63

See: Bergé, A.-M., Martinet, J. \& Olivier, M. . . . . . . 869

Generalized noninterpolatory rules for Cauchy principal value integrals ............... 271

Quadrature formulae and functions of exponential type . 245

A finite element method for time-dependent convection-

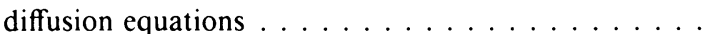

Rigorous sensitivity analysis for systems of linear and non-

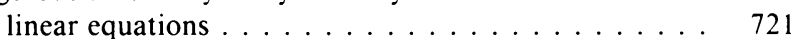

See: Buchmann, J., Sands, J. W. \& Williams, H. C. . . 855

See: Rahman, Qazi I. \& Schmeisser, Gerhard . . . . . 245

Finite element interpolation of nonsmooth functions satisfying boundary conditions ............ 483

See: Baur, Gerhard \& Shawyer, Bruce . . . . . . . 661

New algorithms for finding irreducible polynomials over

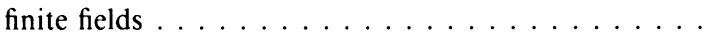

See: Cockburn, Bernardo, Hou, Suchung \& Shu, ChiWANG ................... 545

See: Montgomery, Peter L. \& Silverman, Robert D. .

Lattice rules: projection regularity and unique representations . . . . . . . . . . . . . . . . . 649

A computer search of rank-2 lattice rules for multidimensional quadrature . . . . . . . . . . . . . 281

See: Niederreiter, Harald \& Sloan, Ian H. . . . . . . 303

Error analysis of some finite element methods for the Stokes

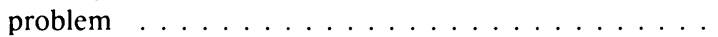

See: Hasegawa, Takemitsu, Toril, Tatsuo \& Sugiura,

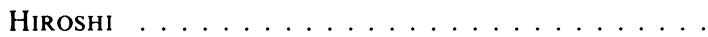

On the stability and convergence of higher-order mixed finite element methods for second-order elliptic prob-

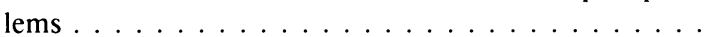

See: Johnson, Claes, Szepessy, Anders \& Hansbo, Peter .................... 107

See: Wei, Yuan \& TaO, TANG . . . . . . . . . . 155

The computational complexity of the resolution of plane curve singularities .............. 797

See: Edixhoven, Bas, de Groot, ARnold \& Top, JaAP . 413

See: Hasegawa, Takemitsu, Toril, Tatsuo \& Sugiura, Hiroshi ................... 195

A note on discrete solutions of the Plateau problem . . . 131

See: Bruaset, A. M., Tveito, A. \& Winther, R. . . . . 701

Symmetrizable finite difference operators . . . . . . . 525

See: Hanlon, Phil \& Wales, David . . . . . . . . 771

See: Sloan, Ian H. \& Walsh, Linda . . . . . . . . 281

Factoring multivariate polynomials over large finite fields. 755 
Author

Wei, Yuan \& TaO, TANG

WILLIAMS, H. C.

WINTHER, R.

YAN, Y $Y_{1}$

ZhaNG, Shangyou
Title

Page

The numerical analysis of implicit Runge-Kutta methods for a certain nonlinear integro-differential equation . . 155

See: Buchmann, J., Sands, J. W. \& Williams, H. C. . . 855

See: Bruaset, A. M., Tveito, A. \& Winther, R. . . . . 701

The collocation method for first-kind boundary integral equa-

tions on polygonal regions. . . . . . . . . . . . . . 139

See: Scott, L. Ridgway \& ZhaNG, SHANGyou . . . . 483 